\title{
In situ XRM Observation of Cracking in CFRP during Nanomechanical Testing
}

\author{
Toshiki Watanabe $^{1, *}$, Yasuo Takeichi ${ }^{1,2}$, Yasuhiro Niwa ${ }^{1}$, and Masao Kimura ${ }^{1,2}$ \\ 1. Inst. of Materials Structure Science, High Energy Accelerator Research Organization (KEK), \\ Tsukuba, Japan. \\ 2. Dept. Mater. Structure Sci., School of High Energy Accelerator Sci., SOKENDAI, Tsukuba, Japan. \\ *Corresponding Author: toshiki.watanabe@kek.jp
}

Carbon fiber reinforced polymer (CFRP) composites are of growing use in aircraft because of their high specific strength and stiffness. Crack initiation and propagation determine mechanical properties of CFRP, though their mechanism has not been fully understood because of experimental difficulties. X-ray computed tomography (X-CT) is a powerful tool to understand the micromechanism. Recent reports pioneered the micromechanical analysis of fractures under quasi-static stress [1] and fatigue failures [2] in CFRP from three-dimensional dataset obtained using synchrotron X-CT. However, most studies deal with large specimens with a special resolution larger than a $\mu \mathrm{m}$.

In order to reveal the micromechanism of crack initiation and propagation, we have performed X-CT measurements with a resolution down to $50 \mathrm{~nm}$ using in situ XRM system. X-ray images were collected using Zernike phase contrast with phase ring because CFRP is composed of low contrast components: fiber and polymer.

In situ mechanical testing has been developed for X-CT measurements with a mechanical stress applied: indentation with a diamond apex to initiate cracks or tensile testing. The key was to optimize experimental conditions such as sample positioning and nanomechanical testing, and procedure of image analysis. These are especially challenging tasks in the case of X-CT with the high resolution. We combined the developed technique with a new XRM installed at the NW2A beamline of a synchrotron radiation facility: PF-AR, IMSS, KEK in Japan.

The nanomechanical testing apparatus features a high precision piezo actuator and an integrated load cell (Figure 1a), enabling the load-displacement curve to be measured during X-CT measurements. Figure $1 \mathrm{~b}$ shows a schematic of the diamond indentation; a cylindrical specimen $\left(c a .60 \mu \mathrm{m}^{\mathrm{D}} \times 150\right.$ $\mu \mathrm{m}^{\mathrm{L}}$ ) was indented with the diamond apex to initiate and propagate cracks. Transparent X-ray images were collected during the indentation with a time interval of $1 \mathrm{sec}$., and the slight but significant change in images, caused by the crack propagation, was clearly observed. Change in the images was visualized by differentiating the serial images, and it was found that the point of large dilatation, corresponding to the crack tip, moved from the indentation side to the virgin side. This shows that an external stress was successfully applied to the specimen using the system of nanomechanical testing. Then, we maintained the amount of indentation and performed in situ X-CT measurements. Typical conditions are: -75 to +75 degree rotation with a one degree step, the exposure time of 10 seconds at each angle, and the total measurement time of 25 minutes.

Figure 2 shows typical examples of cross section images of the three-dimensional volume data reconstructed from 151 projection images. Interfaces between carbon fibers and epoxy resin were clearly observed. It should be noted the images show features not the surface but inside the specimen and that the crack initiation and propagation is free from surface effects. Crack branching in resin observed in lower slices (in small-strain area), whereas cracks propagate along the interface between carbon fibers and epoxy resin in upper ones (in large-strain area) 
We have successfully developed the technique of nanomechanical testing for in situ X-CT observation of crack propagation in CFRP with XRM using synchrotron radiation. The obtained information is inevitable to understand the micromechanism of crack initiation and propagation in CFRP, and being used to R\&D of new types of CFRP [3,4].

\section{References:}

[1] A. E. Scott et al, Compos. Sci. Technol., 71 (2011), p. 1471.

[2] S. C. Garcea et al, Compos. Sci. Technol., 109 (2015), p. 32.

[3] Structural Materials for Innovation of the Cross ministerial Strategic Innovation Promotion Program (SM ${ }^{4}$ I, SIP) of Japan Science and Technology Agency(JST).

[4] Photon Factory at IMSS, KEK Program Advisory Committee (Proposal Nos. 2015S2-002 and 2016S2-001).

(a)

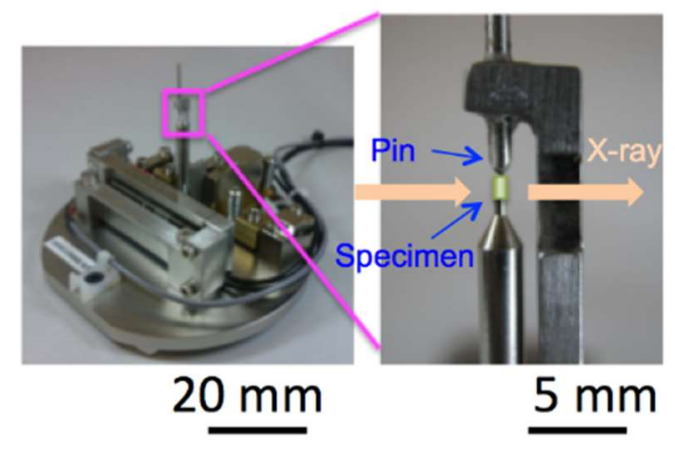

(b)

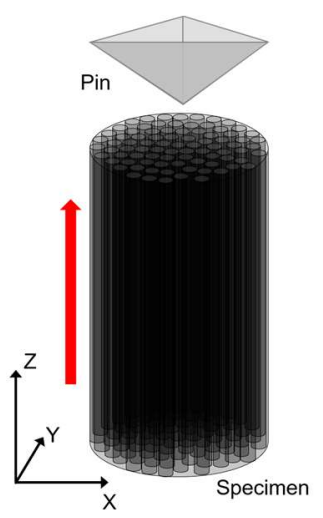

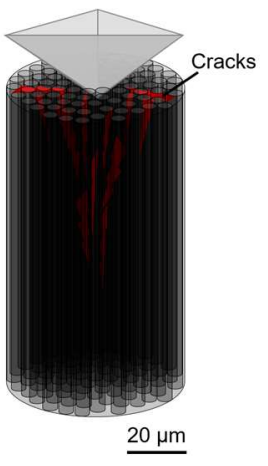

$\underline{20 \mu \mathrm{m}}$

Figure 1. Nanomechanical testing stage. (a) pictures and (b) schematic of the diamond indentation.

(a)

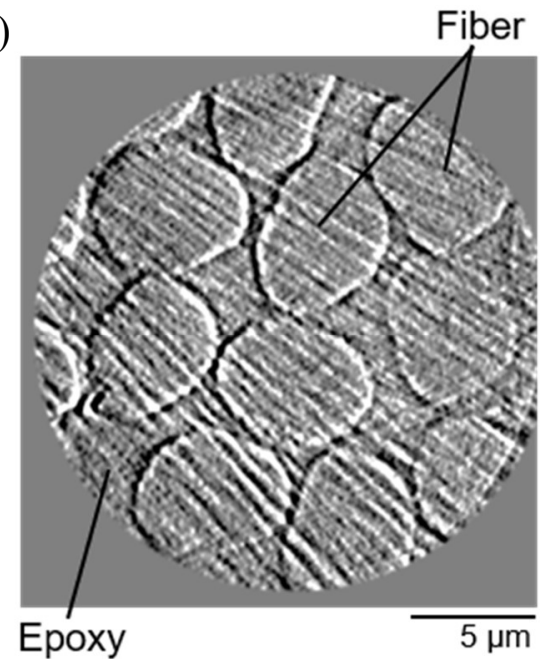

(b)

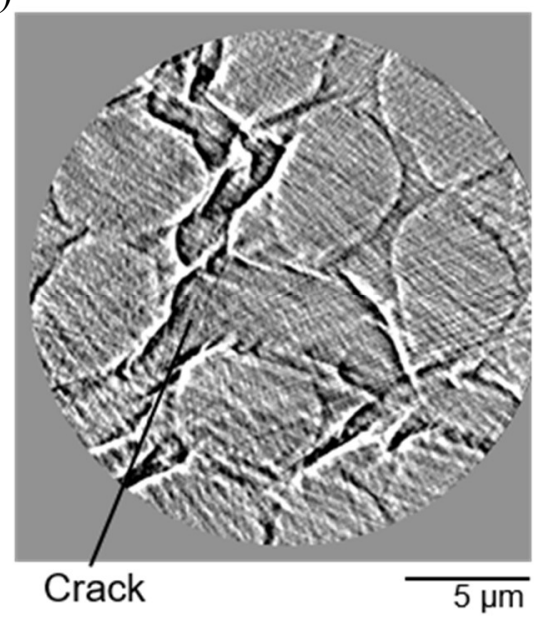

(c)

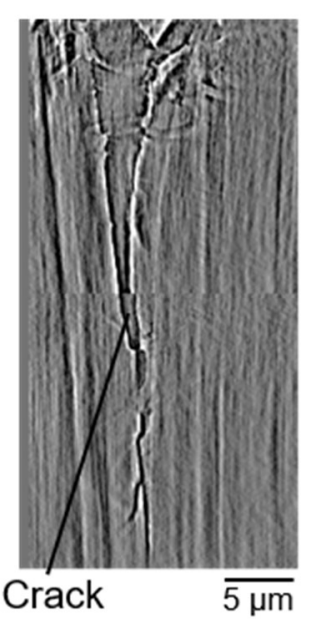

Figure 2. A cross section image of the volume data obtained by X-CT after the crack propagation. (a) XY bottom slice, (b) XY upper slice, and (c) XZ slice. 